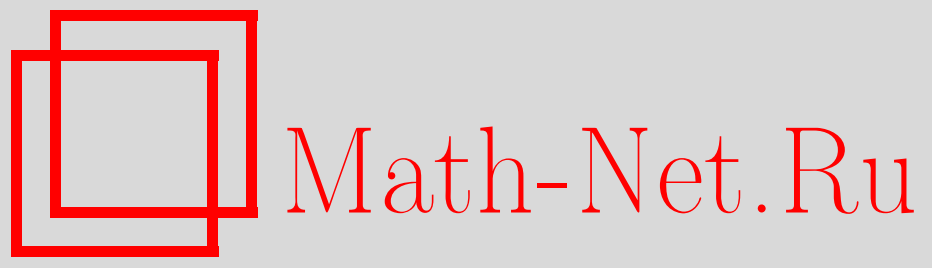

L. Li, From Lyapunov functions to Sobolev inequalities, Теория вероятн. и ее примен., 2014, том 59, выпуск 4, 808-814

DOI: https://doi.org/10.4213/tvp4599

Использование Общероссийского математического портала Math-Net.Ru подразумевает, что вы прочитали и согласны с пользовательским соглашением http: //www . mathnet.ru/rus/agreement

Параметры загрузки:

IP: 34.239 .49 .27

26 апреля 2023 г., 10:50:43

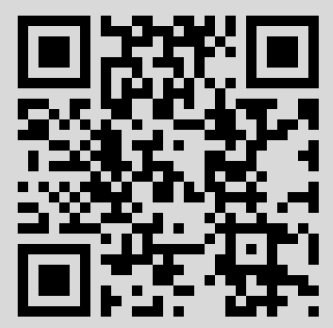




\title{
FROM LYAPUNOV FUNCTIONS TO SOBOLEV INEQUALITIES ${ }^{1)}$
}

\author{
С использованием функций Ляпунова рассмотрены слабые \\ и локальные неравенства типа $\Psi$-Соболева, обеспечивающие \\ скорость сходимости группы.
}

Ключевые слова и фразы: функции Ляпунова, неравенства $\Psi$ Соболева, скорость сходимости, полугруппы.

1. Introduction. The functional inequalities (Poincaré inequality, entropy inequality, logarithmic Sobolev inequality) turned to be the powerful tools for solving the various problems in probability theory and statistical physics.

In recent years, rate of convergence to equilibrium is mostly connected to the above functional inequalities. For instance, Poincaré inequality describes the exponential decay of the semigroup in the $\mathbf{L}^{2}$ sense. Algebraic convergence of transition function to equilibrium in the $\mathbf{L}^{2}$ sense have been studied for the various Markov chains and interacting particle systems (see [7], [5], [4]). Röckner and Wang [9] introduced a weak Poincaré inequality to describe general convergence rates of the semigroup. Cattiaux, Gentil, and Guillin [3] described a weak logarithmic Sobolev inequalities which is used for deriving entropic convergence. Gao and $\mathrm{Li}$ [6] gave a criterion of algebraic convergence in the entropy sense and introduced the weak entropy inequality for describing its relationship to the entropic convergence. Bakry, Cattiaux, and Guillin [1] studied the relationship between the Lyapunov type controls and functional inequalities for quantitative ergodic properties. Now, the new Lyapunov type inequalities will be studied for estimating the convergence rate of semigroup.

In this paper, Let $(E, \mathscr{F}, \mu)$ be a probability space and $\left(X_{t}\right)_{t \geqslant 0}$ be a Markov process on $E$. We denote by $\mathscr{L}$ the infinitesimal generator and $D(\mathscr{L})$ the domain of the generator. $P_{t}$ (respectively, $P_{t}^{*}$ ) is the associated Markov semigroup (respectively, the dual semigroup).

It is known that Meyn and Tweedie [8] deeply developed the stochastic stability. One of the most important results is the existence of the Lyapunov function which yields exponential convergence and the excursions of the process. The definition of the Lyapunov function is firstly presented.

D e f i n it i o n 1.1 (see [8]). Assume that $\phi$ is a positive function defined on $[1,+\infty)$. For $V \in D(\mathscr{L}), V$ is called a $\phi$-Lyapunov function if $V \geqslant 1$ and if there is $b>0$ and a closed petite set $C$ such that

$$
\mathscr{L} V \leqslant-\phi(V)+b 1_{C} .
$$

$\mathrm{R}$ e $\mathrm{m}$ a $\mathrm{r} \mathrm{k}$ 1.1. $C$ is a petite set if there exists some probability measure $\alpha(d t)$ on $\mathbf{R}^{+}$such that for all $x \in C, \int_{0}^{+\infty} P_{t}(x, \cdot) \alpha(d t) \geqslant \nu(\cdot)$, where $\nu(\cdot)$ is a nontrivial positive measure and $P_{t}(x, \cdot)$ is the $\mathbb{P}_{x}$ law of $X_{t}$.

Let $\mu$ be an invariant measure for Markov process with the generator $\mathscr{L}$, that is

$$
E_{\mu}(\mathscr{L} f)=0 \quad \forall f \in D(\mathscr{L}) .
$$

* Department of Mathematics, Tongji University, Shanghai 200092, P.R.China; e-mail: li3nali@tongji.edu.cn

1) The work is supported by the National Natural Science Foundation of China (grant № 10926115). 
The main hypothesis is the existence of the «carré du champ» operator $\Gamma$. We assume that there is an algebra $\mathscr{A} \subset D(\mathscr{L})\left(\mathscr{A}\right.$ is dense in $D(\mathscr{L})$ and $\left.\mathbf{L}^{2}(\mu)\right)$ such that for $f, g \in \mathscr{A}$

$$
\Gamma(f, g)=\mathscr{L}(f g)-f \mathscr{L} g-g \mathscr{L} f
$$

and for $f, g, h \in \mathscr{A}$

$$
\Gamma(f g, h)=f \Gamma(g, h)+g \Gamma(f, h) .
$$

We also show that for all smooth $\Psi$ and $f \in \mathscr{A}$,

$$
\mathscr{L} \Psi(f)=\frac{\partial \Psi}{\partial x}(f) \mathscr{L} f+\frac{1}{2} \frac{\partial^{2} \Psi}{\partial x^{2}}(f) \Gamma(f),
$$

where $\Gamma(f)=\Gamma(f, f)$.

In section 2 , the weak Lyapunov- $\Psi$-Sobolev inequality is discussed for estimating the convergence rate of the semigroup. In section 3, local Lyapunov $\Psi$-Sobolev inequality is presented. The $\mathrm{W}-\mathrm{Lyapunov}-\Psi$-Sobolev inequality and its weak version are deduced from the existence of Lyapunov functions together with some additional assumptions.

2. The weak W-Lyapunov $-\Psi-$ Sobolev inequality. Bakry, Cattiaux, and Guillin [1] showed that the Meyn-Tweedie method immediately furnished some Poincaré inequalities and they studied the relationship between some modified Poincaré inequalities and the existence of a Lyapunov function. They also extended the Poincaré type inequality to the more weak version.

But in many significant cases for physics and information theory, the convergence rate of semigroup in the entropy sense is more useful. Then they described the more general functional inequalities such as $W$-Lyapunov- $\Psi$-Sobolev inequality.

D e f i n i t i o n 2.1 (see [1]). Assume that $\Psi$ is a nonnegative function and $\Psi(1)=$ 0 . $\mu$ satisfies a $W$-Lyapunov- $\Psi$-Sobolev inequality if and only if there exists $W \in D(\mathscr{L})$ with $W \geqslant 1$ and a constant $C_{\Psi}$ for all nice nonnegative $f$ with $\int f d \mu=1$,

$$
\int \Psi(f) W d \mu \leqslant C_{\Psi} \int\left(\frac{1}{2} W \Psi^{\prime \prime}(f) \Gamma(f)-\Psi(f) \mathscr{L} W\right) d \mu .
$$

Here, «nice $f \gg$ means that $f$ is smooth and $f \in D(\mathscr{L}) \cap \mathbf{L}_{2}(\mu)$. Based on the above definition, the following statements are equivalent (see [1]):

(i) $\mu$ satisfies a $W$-Lyapunov- $\Psi$-Sobolev inequality,

(ii) $\int \Psi\left(P_{t}^{*} f\right) W d \mu \leqslant e^{-\left(t / C_{\Psi}\right)} \int \Psi(f) W d \mu$ for all nonnegative $f$ with $\int f d \mu=1$.

That implies that $W$-Lyapunov- $\Psi$-Sobolev inequality describes the exponential decay of semigroup. The main aim of this section is to introduce a general version of (2.1) to describe more general convergence rates of $P_{t}$. Then as the extension of (2.1), the weak $W$-Lyapunov- $\Psi$-Sobolev inequality is presented.

$\mathrm{D}$ e f i n i t i o n 2.2. Assume that $\Psi$ is a nonnegative function and $\Psi(1)=0$. Let $\Phi$ be a nonnegative function on $\mathbf{L}^{2}(\mu)$. $\mu$ satisfies a weak $W$-Lyapunov- $\Psi$-Sobolev inequality if and only if there exists $W \in D(\mathscr{L})$ with $W \geqslant 1$ and a constant $C_{\Psi}$ and a function $\beta$ such that for all nice nonnegative $f$ with $\int f d \mu=1$,

$$
\int \Psi(f) W d \mu \leqslant C_{\Psi} \beta(r) \int\left(\frac{1}{2} W \Psi^{\prime \prime}(f) \Gamma(f)-\Psi(f) \mathscr{L} W\right) d \mu+r \Phi(f),
$$

where $\beta$ is a nonnegative and decreasing function on $(0,+\infty)$.

Then we can establish the relationship between (2.2) and the convergence rate of $P_{t}$.

Theorem 2.1. Assume that (2.2) holds and $\mathscr{L}$ is symmetric with respect to $\mu$. Then for $f$ with $\int f d \mu=1$ and $t>0$,

$$
\int \Psi\left(P_{t} f\right) W d \mu \leqslant \inf _{r>0}\left\{r \sup _{s \in[0, t]} \Phi\left(P_{s} f\right)+\exp \left(-\frac{t}{C_{\Psi} \beta(r)}\right) \int \Psi(f) W d \mu\right\} .
$$


Furthermore, if $\Phi\left(P_{t} f\right) \leqslant \Phi(f)(t>0)$, then

$$
\int \Psi\left(P_{t} f\right) W d \mu \leqslant \eta(t)\left[\Phi(f)+\int \Psi(f) W d \mu\right],
$$

where $\eta(t):=\inf \left\{r>0:-C_{\Psi} \beta(r) \ln r \leqslant t\right\}(t>0)$.

P r o o f. Set $I(t)=\int \Psi\left(P_{t} f\right) W d \mu \geqslant 0$. Because $\mu$ is invariant, applying (2.2) with $P_{t} f$, we thus get

$$
\begin{aligned}
I^{\prime}(t) & =\int \Psi^{\prime}\left(P_{t} f\right) \mathscr{L}_{t} f W d \mu \\
& =\int\left[-\frac{1}{2} \Psi^{\prime \prime}\left(P_{t} f\right) \Gamma\left(P_{t} f\right)+\mathscr{L}\left(\Psi\left(P_{t} f\right)\right)\right] W d \mu \\
& \leqslant-\frac{1}{C_{\Psi} \beta(r)} \int \Psi\left(P_{t} f\right) W d \mu+\frac{r}{C_{\Psi} \beta(r)} \Phi\left(P_{t} f\right) \\
& =-\frac{1}{C_{\Psi} \beta(r)}\left(I(t)-r \Phi\left(P_{t} f\right)\right) .
\end{aligned}
$$

If there exist $t_{0}>0$ and $r>0$ such that

$$
\int \Psi\left(P_{t_{0}} f\right) W d \mu>r \sup _{s \in\left[0, t_{0}\right]} \Phi\left(P_{s} f\right)+\exp \left[-\frac{t_{0}}{C_{\Psi} \beta(r)}\right] \int \Psi(f) W d \mu .
$$

Then for all $0 \leqslant t \leqslant t_{0}, I(t) \geqslant I\left(t_{0}\right)>r \sup _{s \in\left[0, t_{0}\right]} \Phi\left(P_{s} f\right)$ and so by $(2.5)$

$$
\frac{I^{\prime}(t)}{I(t)-r \sup _{s \in\left[0, t_{0}\right]} \Phi\left(P_{s} f\right)} \leqslant-\frac{1}{C_{\Psi} \beta(r)} .
$$

Which implies that for all $t \in\left[0, t_{0}\right]$,

$$
I(t)-r \sup _{s \in\left[0, t_{0}\right]} \Phi\left(P_{s} f\right) \leqslant \exp \left[-\frac{t}{C_{\Psi} \beta(r)}\right]\left(I(0)-r \sup _{s \in\left[0, t_{0}\right]} \Phi\left(P_{s} f\right)\right) .
$$

Thus, for all $t \in\left[0, t_{0}\right]$,

$$
\int \Psi\left(P_{t} f\right) W d \mu \leqslant r \sup _{s \in\left[0, t_{0}\right]} \Phi\left(P_{s} f\right)+\exp \left[-\frac{t}{C_{\Psi} \beta(r)}\right] \int \Psi(f) W d \mu,
$$

which is a contradiction to (2.5). Consequently, the result (2.3) is valid. Furthermore, if $\Phi\left(P_{t} f\right) \leqslant \Phi(f)$, then

$$
\int \Psi\left(P_{t} f\right) W d \mu \leqslant \inf _{r>0}\left\{r \Phi(f)+\exp \left[-\frac{t}{C_{\Psi} \beta(r)}\right] \int \Psi(f) W d \mu\right\} .
$$

Define $\eta(t)=\inf \left\{r>0:-C_{\Psi} \beta(r) \ln r \leqslant t\right\}(t>0)$, that yields (2.4). Theorem 2.1 is proved.

E x a m p l e 2.1. Consider the Ornstein-Uhlenbeck operator $\mathscr{L}:=\triangle-x \cdot \nabla$ on $\mathbf{R}^{d}$. Then $\Gamma(f)=\int|\nabla f|^{2} d \mu$, where $\mu(d x):=Z^{-1} e^{-|x|^{2} / 2} d x$ with $Z>0$ the normalization. By [10, Remark 2.2], one has

$$
\int f^{2} d \mu \leqslant \frac{c}{\ln r-c} \int|\nabla f|^{2} d \mu+r\left(\int|f| d \mu\right)^{2}, \quad r \geqslant e^{2 c},
$$

for some $c>0$. If we choose $\Psi(f)=f(f-1) \mathbf{1}_{\{f \geqslant 1\}}+f^{2} \mathbf{1}_{\{f<1\}}$, then (2.2) holds for $W=1, \beta(r)=c / \ln (r-c), r \geqslant e^{2 c}$, and $\Phi(f)=\left(\int|f| d \mu\right)^{2}$. Since $\mu$ is an invariant measure, it is obvious that $\Phi\left(P_{t} f\right) \leqslant \Phi(f)$. 
Furthermore, assume

$$
\int_{1}^{+\infty} \frac{\beta(r)}{r} d r<+\infty
$$

We can improve Theorem 2.1.

Theorem 2.2. If (2.6) holds and $\Phi\left(P_{t} f\right) \leqslant \Phi(f)$, let

$$
\zeta(t)=\int_{t}^{+\infty} \frac{\beta(r)}{r} d r, \quad t>0 .
$$

Then (2.2) implies

$$
\int \Psi\left(P_{t} f\right) W d \mu \leqslant \eta(t) \Phi(f), \quad f \in D(\mathscr{L})_{+}, \quad \int f d \mu=1,
$$

for $\eta(t)=\inf _{\varepsilon \in(0,1)} \varepsilon^{-1} \zeta^{-1}\left((1-\varepsilon) t / C_{\Psi}\right)$.

P r o o f. For any $f \in D(\mathscr{L})_{+}$with $\int f d \mu=1$ and $\Phi(f)=1$, define $I(t)=$ $\int \Psi\left(P_{t} f\right) W d \mu$. By the weak $W$-Lyapunov- $\Psi$-Sobolev inequality $(2.2)$ with $C_{\Psi}>0$ and $\Phi\left(P_{t} f\right) \leqslant \Phi(f)$, we get

$$
I^{\prime}(t) \leqslant-\frac{I(t)}{C_{\Psi} \beta(r)}+\frac{r}{C_{\Psi} \beta(r)} .
$$

Taking $r=\varepsilon I(t)$, we have

$$
I^{\prime}(t) \leqslant-\frac{(1-\varepsilon) I(t)}{C_{\Psi} \beta(\varepsilon I(t))}
$$

Therefore,

$$
\frac{-(1-\varepsilon) t}{C_{\Psi}} \geqslant \int_{0}^{t} \frac{I^{\prime}(s) \beta(\varepsilon I(s))}{I(s)} d s=\int_{\varepsilon I(0)}^{\varepsilon I(t)} \frac{\beta(r)}{r} d r \geqslant-\zeta(\varepsilon I(t)) .
$$

This implies that $I(t) \leqslant \varepsilon^{-1} \zeta^{-1}\left((1-\varepsilon) t / C_{\Psi}\right)$. Theorem 2.2 is proved.

$\mathrm{R}$ e $\mathrm{m}$ a r k 2.1. (1) Suppose that $\mu$ satisfies a weak $W$-Lyapunov- $\Psi$-Sobolev inequality (2.2) and $\Phi\left(P_{t} f\right) \leqslant \Phi(f)$. For all $r \in[0,1 / 2]$, some $\delta \in(0,1)$ and $c>0$, if $\beta(r)=c\left(\ln r^{-1}\right)^{(1-\delta) / \delta}$, then $(2.7)$ holds for

$$
\eta(t)=c_{1} t \exp \left\{-\left(\frac{t}{\delta c}\right)^{\delta}\right\} \text { for some } c_{1}>0 .
$$

This implies the subexponential convergence.

(2) Assume that $\mu$ satisfies a weak $W$-Lyapunov- $\Psi$-Sobolev inequality (2.2) and $\Phi\left(P_{t} f\right) \leqslant \Phi(f)$. For all $r>0$, some $c>0$ and $p>1$, if $\beta(r)=c r^{1-p}$, then $(2.7)$ holds for

$$
\eta(t)=q^{q}\left(\frac{t}{c_{2}}\right)^{1-q}, \quad \frac{1}{p}+\frac{1}{q}=1 \text { and some } c_{2}>0 .
$$

This means the algebraic convergence.

3. The local $\Psi$-Sobolev inequality. In order to find the sufficient conditions for the Lyapunov-Poincaré inequality to hold, Bakry, Cattiaux, and Guillin (see [1]) gave the local Lyapunov-Poincaré inequality. With the existence of Lyapunov functions and some assumptions, they deduced the $W$-Lyapunov-Poincaré inequality. For Lyapunov$\Psi$-Sobolev inequality, they did not introduce the local version of some new $\Psi$-Sobolev inequalities but focused on a typical example. Now we turn to find some Lyapunov conditions for the (weak) $W$-Lyapunov- $\Psi$-Sobolev inequality to hold which in turn yield the convergence rate of $P_{t}$. Recall the definition (1.1) of Lyapunov function $V$, we have to assume that $\int V d \mu<+\infty$. Similar to the local Poincaré inequality, we shall introduce the following definition. 
D e f i n i t i o n 3.1. We shall say that $\mu$ satisfies a local $\Psi$-Sobolev inequality on $U(U \subset E)$ if there exists some constant $\gamma_{U}$ such that for all nice $\mathrm{f}$ with $\int_{E} f d \mu=1$,

$$
\int_{U} \Psi(f) d \mu \leqslant \gamma_{U} \int_{E} \frac{1}{2} \Psi^{\prime \prime}(f) \Gamma(f) d \mu+\frac{1}{\mu(U)} \Psi\left(\int_{U} f d \mu\right) .
$$

Theorem 3.1. Assume that there exists a Lyapunov function $V$, i.e., $\mathscr{L} V \leqslant-2 \alpha V+$ $b \mathbf{1}_{C}$ on some set $C$. Suppose that one can find a set $U$ such that $\mu$ satisfies a local $\Psi$ Sobolev inequality on $U$. Furthermore, we assume that $U$ contains $C^{\prime}=C \cap\{V \leqslant b / \alpha\}$ and $\alpha \mu(U)>b \mu\left(U^{c}\right)$. In addition, we suppose that $\Psi\left(\int_{U} f d \mu\right) \leqslant \mu\left(U^{c}\right) \int \Psi(f) d \mu$. Then we can find some $\lambda>0$ such that if $W=V+\lambda$, $\mu$ satisfies a $W$-Lyaponov- $\Psi$-Sobolev inequality.

P r o o f. Obviously, for $C^{\prime}=C \cap\{V \leqslant b / \alpha\}$, we have $\mathscr{L} V \leqslant-\alpha V+b \mathbf{1}_{C^{\prime}}$. For any $\lambda>0$, with $V \geqslant 1$ and $\Psi(f) \geqslant 0$, we obtain

$$
\begin{aligned}
\int \Psi(f)(V+\lambda) d \mu & \leqslant(1+\lambda) \int \Psi(f) V d \mu \\
& \leqslant \frac{1+\lambda}{\alpha} \int \Psi(f)\left(-\mathscr{L}(V+\lambda)+b \mathbf{1}_{C^{\prime}}\right) d \mu .
\end{aligned}
$$

From the local $\Psi$-Sobolev inequality (3.1) and $\Psi\left(\int_{U} f d \mu\right) \leqslant \mu\left(U^{c}\right) \int \Psi(f) d \mu$, it holds that

$$
\begin{aligned}
\int_{C^{\prime}} \Psi(f) d \mu & \leqslant \int_{U} \Psi(f) d \mu \\
& \leqslant \gamma_{U} \int \frac{1}{2} \Psi^{\prime \prime}(f) \Gamma(f) d \mu+\frac{1}{\mu(U)} \Psi\left(\int_{U} f d \mu\right) \\
& \leqslant \gamma_{U} \int \frac{1}{2} \Psi^{\prime \prime}(f) \Gamma(f) d \mu+\frac{\mu\left(U^{c}\right)}{\mu(U)} \int \Psi(f) V d \mu .
\end{aligned}
$$

Let $\lambda=\left(b \gamma_{U}-1\right)_{+}$. We get

$$
\begin{aligned}
b \int_{C^{\prime}} \Psi(f) d \mu & \leqslant \int \frac{1}{2} \Psi^{\prime \prime}(f) \Gamma(f)\left(\left(b \gamma_{U}-1\right)+1\right) d \mu+\frac{b \mu\left(U^{c}\right)}{\mu(U)} \int \Psi(f) V d \mu \\
& \leqslant \int \frac{1}{2} \Psi^{\prime \prime}(f) \Gamma(f)(V+\lambda) d \mu+\frac{b \mu\left(U^{c}\right)}{\mu(U)} \int \Psi(f) V d \mu .
\end{aligned}
$$

Combining the second inequality in (3.3) and (3.4), we have

$$
\begin{aligned}
\int\left(\frac{1}{2} \Psi^{\prime \prime}(f) \Gamma(f) W-\Psi(f) \mathscr{L} W\right) d \mu & \geqslant\left(\alpha-\frac{b \mu\left(U^{c}\right)}{\mu(U)}\right) \int \Psi(f) V d \mu \\
& \geqslant \frac{\alpha}{1+\lambda}\left(1-\frac{b \mu\left(U^{c}\right)}{\alpha \mu(U)}\right) \int \Psi(f) W d \mu .
\end{aligned}
$$

Then $\mu$ satisfies a $W$-Lyapunov- $\Psi$-Sobolev inequality with $W=V+\lambda$ and $1 / C_{\Psi}=$ $\alpha /(1+\lambda)\left(1-b \mu\left(U^{c}\right) / \alpha \mu(U)\right)$. Theorem 3.1 is proved.

Similarly, we shall extend the above theorem to weak framework.

Theorem 3.2. Assume that there exists a $2 \phi$-Lyapunov function $V$, i.e., $\mathscr{L} V \leqslant$ $-2 \phi(V)+b \mathbf{1}_{C}$ on some set $C$ and $\phi(u) \geqslant M>0$. Suppose that one can find a set $U$ such that $\mu$ satisfies a local $\Psi$-Sobolev inequality on $U$. Furthermore, we assume that $U$ contains $C^{\prime}=C \cap\{\phi(V) \leqslant b\}$ and $M \mu(U)>b \mu\left(U^{c}\right)$. In addition, we suppose that $\Psi\left(\int_{U} f d \mu\right) \leqslant \mu\left(U^{c}\right) \int \Psi(f) d \mu$. Then we can find some $\lambda>0$ such that $\mu$ satisfies a weak $W$-Lyapunov- $\Psi$-Sobolev inequality, i.e., for all $f$ with $\int f d \mu=1$ and $r>0$,

$$
\int \Psi(f) W d \mu \leqslant D_{\Psi} \beta(r)\left(\int\left(\frac{1}{2} \Psi^{\prime \prime}(f) \Gamma(f) W-\Psi(f) \mathscr{L} W\right) d \mu\right)+r\|\Psi(f)\|_{\infty},
$$


where $W=V+\lambda, \beta(r)=\inf \left\{u ; \int_{V>u \phi(V)} V d \mu \leqslant r\right\}$ and $D_{\Psi}=(1+\lambda) /(1-$ $\left.b \mu\left(U^{c}\right) / M \mu(U)\right)$.

P r o o f. As in the proof of Theorem 3.1, we have

$$
\int \Psi(f)(V+\lambda) d \mu \leqslant(1+\lambda) \int \Psi(f) V d \mu .
$$

Notice that

$$
\begin{aligned}
\int \Psi(f) V d \mu & \leqslant u \int_{V \leqslant u \phi(V)} \Psi(f) \phi(V) d \mu+\|\Psi(f)\|_{\infty} \int_{V \geqslant u \phi(V)} V d \mu \\
& \leqslant \beta(r) \int \Psi(f) \phi(V) d \mu+r\|\Psi(f)\|_{\infty} .
\end{aligned}
$$

Recalling the existence of the $\phi$-Lyapunov function (1.1), we get

$$
\int \Psi(f) \phi(V) d \mu \leqslant \int \Psi(f)\left(-\mathscr{L}(V+\lambda)+b \mathbf{1}_{C^{\prime}}\right) d \mu .
$$

As in the proof of (3.4), we find

$$
b \int_{C^{\prime}} \Psi(f) d \mu \leqslant \int \frac{1}{2} \Psi^{\prime \prime}(f) \Gamma(f) W+\frac{b \mu\left(U^{c}\right)}{M \mu(U)} \int \Psi(f) \phi(V) d \mu .
$$

Then

$$
\begin{aligned}
\int \Psi(f) \phi(V) d \mu \leqslant & \frac{1}{1-b \mu\left(U^{c}\right) /(M \mu(U))} \\
& \times\left(\int\left(\frac{1}{2} \Psi^{\prime \prime}(f) \Gamma(f) W-\Psi(f) \mathscr{L} W\right) d \mu\right) .
\end{aligned}
$$

From the above formulas, it holds that

$$
\begin{aligned}
\int \Psi(f) W d \mu \leqslant & \frac{1+\lambda}{1-b \mu\left(U^{c}\right) /(M \mu(U))} \beta(r) \\
& \times\left(\int\left(\frac{1}{2} \Psi^{\prime \prime}(f) \Gamma(f) W-\Psi(f) \mathscr{L} W\right) d \mu\right)+r\|\Psi(f)\|_{\infty} .
\end{aligned}
$$

Theorem 3.2 is proved.

Corollary 3.1. In the situation of Theorem 3.2 , for $f$ with $\int f d \mu=1$ and $t>0$,

$$
\int \Psi\left(P_{t} f\right) W d \mu \leqslant \inf _{r>0}\left\{r \sup _{s \in[0, t]}\left\|\Psi\left(P_{s} f\right)\right\|_{\infty}+\exp \left(-\frac{t}{D_{\Psi} \beta(r)}\right) \int \Psi(f) W d \mu\right\} .
$$

Moreover, if $\left\|\Psi\left(P_{t} f\right)\right\|_{\infty} \leqslant\|\Psi(f)\|_{\infty}, t>0$, then

$$
\int \Psi\left(P_{t} f\right) W d \mu \leqslant \eta(t)\left[\|\Psi(f)\|_{\infty}+\int \Psi(f) W d \mu\right],
$$

where $\eta(t):=\inf \left\{r>0:-D_{\Psi} \beta(r) \ln r \leqslant t\right\}, t>0$.

P r o o f. Theorem 2.1 implies the conclusion of this corollary if one takes $\Phi(f)=$ $\|\Psi(f)\|_{\infty}$.

Acknowledgments. It is pleasure to thank the referee for his very valuable report. 


\section{REFERENCES}

1. Bakry D., Cattiaux P., Guillin A. Rate of convergence for ergodic continous Markov processes: Lyapunov versus Poincaré. - J. Funct. Anal, 2008, v. 254, № 3, p. 727-759.

2. Bakry D., Emery M. Hypercontractivité de semi-groups de diffuision. - C. R. Acad. Sci. Paris. Sér. I Math., 1984, v. 299, p. 775-778.

3. Cattiaux P., Gentil I., Guillin A. Weak logarithmic Sobolev inequalities and entropic convergence. - Probab. Theory Related Fields, 2007, v. 139, p. 563-603.

4. Chen M. F., Wang Y.Z. Algebraic convergence of Markov chains. - Ann. Appl. Prob. 2003, v. 13, p. 604-627.

5. Deuschel J. D. Algebraic $L^{2}$ decay of attractive critical processes on the lattic. - Ann. Probab., 1987, v. 15, p. 780-799.

6. Gao F. Q., Li L. N. Weak entropy inequalities and entropic convergence. - Science in China (A), 2008, v. 51, № 10, p. 1798-1806.

7. Liggett T.M. $L^{2}$ rates of convergence of attractive reversible nearst paticle systems: The critical case. - Ann. Probab., 1991, v. 19, p. 935-959.

8. Meyn S.P., Tweedie R.L. Markov Chains and Stochastic Stability. London: SpringVerlag, 1993.

9. Röckner M., Wang F. Y. Weak Poincaré inequalities and $L^{2}$-convergence rates of markov semigroup. - J. Funct. Anal., 2001, v. 185, p. 564-603.

10. Wang F. Y., Zhang Q. Z. Weak Poincaré inequalities, decay of Markov semigroups and concentration of Measures. - Acta Mathematica Sinica, 2005, v. 21, № 4, p. 937-942.

Поступила в редакцию 12.IX.2012

Исправленный вариант

28.XII.2012

(c) $2014 \mathrm{\Gamma}$

PINELIS I.*

\section{ROSENTHAL-TYPE INEQUALITIES FOR MARTINGALES IN 2-SMOOTH BANACH SPACES ${ }^{1}$}

Мы улучшаем ряд известных ранее верхних оценок для моментов норм мартингалов в 2-гладких банаховых пространствах. Некоторые из этих улучшений имеют место даже для сумм независимых действительнозначных случайных величин. Приводятся применения к концентрации меры на произведениях пространств для функций, липшицевых по каждой переменной, в том числе применения, касающиеся центральных моментов нормы сумм независимых случайных векторов в произвольном сепарабельном банаховом пространстве.

Ключевые слова и фразы: вероятностные неравенства; оценки моментов; неравенство Розенталя; 2-гладкие банаховы пространства; гильбертовы пространства; мартингалы; суммы независимых случайных величин; концентрация меры; функции, являющиеся липшицевыми по каждой переменной; произведения пространств.

* Department of Mathematical Sciences, Michigan Technological University, Houghton, Michigan, USA; e-mail: ipinelis@mtu.edu

1) Supported by NSA grant H98230-12-1-0237. 